\title{
The Economic Burden of Nocturia on the U.S. Health Care System and Society: A National Health and Nutrition Examination Survey Analysis
}

\author{
Jay Jhaveri, MD, MPH; Marjolaine Gauthier-Loiselle, PhD; \\ Patrick Gagnon-Sanschagrin, MSc; and Eric Q. Wu, PhD
}

\begin{abstract}
BACKGROUND: Nocturia, characterized as waking during the main sleep period to urinate, is a common condition. Persistent nocturia results in sleep fragmentation with deleterious effects on health and well-being. Yet, there are limited data on the economic burden of nocturia in the United States.
\end{abstract}

OBJECTIVE: To assess the association of nocturia with health care resource utilization (HRU), work productivity, and self-rated health while estimating the societal costs of nocturia in the United States in 2017.

METHODS: A retrospective cross-sectional study was conducted using data from the National Health and Nutrition Examination Survey (NHANES; 2005-2006 to 2013-2014). Adults aged $\geq 18$ years (excluding pregnant women) were stratified into individuals with nocturia ( $\geq 2$ voids/night) and individuals without nocturia ( $<2$ voids/night), based on the threshold for clinically significant nocturia. Outcomes were self-reported and included HRU (hospitalizations, outpatient visits); work productivity (weekly hours worked, employment); and current health status. Multivariable regression analyses adjusting for age, race, sex, body mass index, insurance status, education level, alcohol use, smoking status, and self-reported comorbid conditions were used to compare the 2 cohorts, overall and stratified by age group (20-44 years, 45-64 years, and 65+ years) to distinguish the effects on different age groups including the Medicare-aged population. Excess direct health care costs and indirect productivity costs associated with nocturia in the United States were then calculated using a prevalencebased approach and available literature (i.e., nocturia prevalence estimates, aggregated unit costs by HRU type, and average hourly earnings in the United States).

RESULTS: 22,300 individuals were identified, and $24 \%$ had nocturia $(\geq 2$ voids/night). Median age was 55.2 and 43.2 years among individuals with and without nocturia, respectively, and the proportion of males was $43.3 \%$ and $51.3 \%$, respectively. Individuals with nocturia had significantly more HRU, including hospitalizations and outpatient visits, worked significantly fewer hours weekly, and were significantly less likely to be employed when compared with those without nocturia. They were also significantly less likely to report being in very good/excellent health. These comparisons remained statistically significant across age groups. Total excess direct health care costs were $\$ 62.9$ billion (hospitalization: $\$ 47.6$ billion; outpatient: $\$ 15.3$ billion). Total excess indirect productivity costs were $\$ 151.7$ billion. Altogether, costs were estimated at $\$ 214.5$ billion, equivalent to $\$ 3,491$ per individual with nocturia. Individuals aged $20-44$ years incurred $23.5 \%$ of total excess costs, while those aged $45-64$ and $65+$ years incurred $48.2 \%$ and $28.3 \%$, respectively. Sensitivity analyses based on lower prevalence estimates resulted in costs of $\$ 94.0$ billion, while those based on higher prevalence estimates reached up to $\$ 231.1$ billion.

CONCLUSIONS: Nocturia is associated with a substantial economic burden in the United States even when evaluated based on lower prevalence estimates. This study underscores the importance of timely diagnosis and management of nocturia patients to alleviate health-related and economic consequences to patients and society.

J Manag Care Spec Pharm. 2019;25(12):1398-1408

Copyright $\odot 2019$, Academy of Managed Care Pharmacy. All rights reserved.

\section{What is already known about this subject}

- Nocturia, characterized as waking during the main sleep period to pass urine, is a highly prevalent condition affecting up to a quarter of all adults and up to half of older adults aged 65 years and older.

The sleep disruption caused by nocturia has potentially deleterious effects on daytime alertness, health, and well-being of individuals and has been linked to higher risk of comorbid conditions including hypertension, cardiovascular disease, mood disorders, and mortality.

A few studies have reported that nocturia is associated with a high economic burden, but there are no comprehensive assessments of the total direct health care costs and indirect costs associated with nocturia in the United States based on a nationally representative sample.

\section{What this study adds}

Individuals with nocturia ( $\geq 2$ voids/night) had statistically significantly more hospitalizations and outpatient visits, worked fewer hours weekly, were less likely to be employed, and were less likely to report being in very good or excellent health compared with individuals without nocturia.

Based on a large, representative sample of the U.S. population, this study demonstrates that the economic impact of nocturia on the U.S. health care system and society is substantial, with total excess direct health care costs and indirect productivity costs estimated at $\$ 214.5$ billion, equivalent to $\$ 3,491$ per individual with nocturia, with direct costs accounting for $29 \%$ of total costs. This study raises awareness about the substantial economic impact of nocturia on the U.S. health care system and society, highlighting the importance of timely evaluation and effective nocturia management that may help reduce the burden on individuals and society. 
$\mathrm{N}$ octuria is characterized as waking during the main sleep period to pass urine. ${ }^{1}$ It is a highly prevalent condition with an estimated overall point prevalence of $21.1 \%$ in men and $26.6 \%$ in women in the United States. The prevalence of nocturia increases with age, ranging from $11 \%$ to $18 \%$ among those aged $20-45$ years, from $24 \%$ to $30 \%$ among those aged $46-65$ years, and from $43 \%$ to $48 \%$ among those aged older than 65 years. ${ }^{2}$ The condition is highly multifactorial and can occur in the context of urologic conditions or nonurologic metabolic diseases. ${ }^{4}$ Common risk factors include advancing age, higher body mass index, hypertension, diabetes, metabolic syndrome, cardiovascular disease, and cerebrovascular disease. ${ }^{5}$

Nocturia has been consistently associated with increased prevalence of mood disorders, insulin resistance, obesity, and hypertension and increased risk of driving accidents, falls, fractures, and mortality. ${ }^{6-13}$ Additionally, nocturia affects occupational functioning leading to decreased productivity at work and absenteeism, with work impairment increasing with severity of nocturia..$^{14,15}$

Although nocturia is considered to be one of the most bothersome lower urinary tract symptoms (LUTS), it remains an underdiagnosed and underreported condition, since patients may be reluctant to discuss symptoms and seek treatment due to embarrassment and/or lack of awareness of available treatments. ${ }^{4,17}$ Moreover, some clinicians may not be aware of the patient burden associated with nocturia, may consider it to be of minor concern or a normal part of aging, and may not prioritize diagnosis and timely treatment. ${ }^{18}$ To date, only a handful of studies have examined the economic burden of nocturia, including direct and indirect costs. ${ }^{15,19-22}$ These studies focused mainly on costs related to specific aspects associated with nocturia (e.g., falls and fractures) often in the elderly population ${ }^{19,21}$ or were based outside the United States. ${ }^{20,22}$ One California-based study compared differences in health care resource utilization (HRU), health care costs, and productivity in adult patients with differing frequencies of nocturia (i.e., 1 vs. $\geq 2$ voids/night) but did not estimate the overall economic burden of nocturia in the United States. ${ }^{15}$

To the best of our knowledge, the economic burden associated with nocturia on the U.S. health care system and society has not been adequately quantified in a nationally representative population. The purpose of this study was to examine the association of nocturia with HRU, work productivity, and perceived health and then provide estimations on the economic burden associated with nocturia in the United States.

\section{Methods}

\section{Data Source}

Data were obtained from the National Health and Nutrition Examination Survey (NHANES) database, a cross-sectional survey that aims to examine a nationally representative U.S. sample of approximately 5,000 individuals per year. This survey, which combines interviews and physical examination, is designed to assess the health and nutritional status of adults and children. The NHANES interview includes demographic, socioeconomic, dietary, and health-related questions collected by a health representative of NHANES during a home interview, the content of which varies from year to year. Only survey cycles for which the interview included questions related to urinary symptoms were considered for this study (2005-2006 to 2013-2014). On average, 2 weeks after the interview, a health examination is conducted at a NHANES mobile examination center, consisting of medical, dental, and physiological measurements and laboratory tests performed based on age and gender.

The National Center for Health Statistics Research (NCHS) Ethics Review Board (formerly referred to as The Institutional Review Board of the NCHS) approved the NHANES data collection and distribution via the NHANES website for public use. ${ }^{23}$ The publicly available database does not contain any identifiable individual information and is compliant with the Health Insurance Portability and Accountability Act.

To estimate the economic burden associated with nocturia, a targeted literature review was conducted to identify the prevalence of nocturia, the average costs of HRU (i.e., hospitalizations and outpatient visits), and average hourly earnings in the United States. Aggregated unit costs by type of health care resource were obtained from the U.S. Department of Health \& Human Services Agency for Healthcare Research and Quality. ${ }^{24,25}$ Average hourly earnings in the U.S. employed population were obtained from the U.S. Bureau of Labor Statistics. ${ }^{26}$

\section{Study Design and Sample Selection}

A retrospective cohort cross-sectional study design was used to estimate the economic burden of nocturia at the individual level using the NHANES database. Individuals were included in the study if they (a) were aged $\geq 18$ years, (b) were enrolled in the NHANES study between 2005-2006 and 2013-2014, (c) underwent the NHANES home interview and health examination process as previously described, and (d) answered the NHANES questionnaires on kidney conditions and urology, hospital utilization and access to care, medical conditions, and occupation. Pregnant women were excluded.

Eligible individuals were classified into 2 mutually exclusive cohorts based on the clinically significant threshold for nocturia (i.e., 2 voids/night). ${ }^{27}$ More specifically, individuals were considered as having nocturia if they indicated most typically getting up $\geq 2$ times per night to urinate during the past 30 days, while individuals were considered without nocturia if they indicated typically getting up $<2$ times per night to urinate during the past 30 days. 


\section{Measurements and Outcomes}

Demographic and health behavior characteristics were measured on the NHANES interview date and included age, sex, race, education, health insurance, body mass index, alcohol use, and smoking status (i.e., currently smoking, formerly smoking, or never smoked). Self-reported comorbid conditions were measured any time before the interview date and included cancer (including prostate), chronic obstructive pulmonary disease, congestive heart failure, coronary artery disease, stroke, diabetes mellitus, hypertension, and self-reported urinary leakage.

Outcomes included HRU, work productivity, current health status, and economic burden of nocturia (including direct health care and indirect productivity costs). HRU included selfreported number of hospitalization stays and outpatient visits during the 12 months before the NHANES interview. Work productivity included self-reported employment status and number of hours worked during the week before the NHANES interview, with the number of hours worked considered to be zero if the individual did not work during that week. Finally, current health status was measured based on self-reported general health (i.e., poor, fair, good, very good, or excellent) during the NHANES interview.

\section{Economic Burden of Nocturia in the United States}

Direct health care and indirect productivity costs in the United States were obtained by combining the results from the analyses on HRU and work productivity at the individual level using the NHANES database with information derived from the literature on prevalence of nocturia, aggregated unit costs by type of HRU, and average hourly earnings. Population-level costs were calculated using a prevalence-based approach. The prevalence of nocturia overall and by age group in the U.S. population was calculated using age- and sex-specific prevalence estimates reported in Vaughan et al. (2016), ${ }^{2}$ weighted by the proportion of individuals in each age and sex group in the U.S. population (e.g., $50.3 \%$ and $49.7 \%$ of individuals aged 20-44 years were male and female, respectively). ${ }^{28}$ The number of individuals with nocturia in each age group in the U.S. population was derived from the prevalence of nocturia in each age group reported in Vaughan et al. ${ }^{2}$ and the 2017 U.S. population by age group. Given the variability in the prevalence estimates of nocturia in the literature, a range for the economic burden of nocturia was also calculated assuming the minimum and maximum prevalence estimates reported in Bosch et al. (2010). ${ }^{29}$

Direct health care costs associated with nocturia were estimated based on excess HRU estimated from our analyses using the NHANES database and average HRU costs obtained from available U.S government statistics. ${ }^{24,25}$ The annual excess HRU (i.e., number of hospitalizations and outpatient visits) incurred by individuals with nocturia compared with individuals without nocturia in each age group was first calculated using age group-specific incident rate ratios (IRRs) and the average annual number of medical visits per individual in each age group from our NHANES analyses. The excess direct health care costs per individual in each age group were estimated by applying the average costs of a hospitalization and an outpatient visit across the U.S. population to the estimated excess HRU by age group.

The average costs of a hospitalization, derived from the literature, were reported as the costs a hospital incurs to provide care and included costs for scheduled and emergency stays. ${ }^{25}$ The average costs of an outpatient visit, also derived from the Healthcare Cost and Utilization Project, ${ }^{24}$ included the sum of payments from all sources (e.g., individual, private insurance policy, Medicaid, Medicare, and worker's compensation) and considered office-based, hospital outpatient department, and hospital emergency room visits without an overnight stay. ${ }^{24}$ Direct health care costs associated with nocturia at a population level were calculated from individual level direct health care costs by age group and the estimated number of individuals with nocturia in the U.S. population for each age group.

Indirect productivity costs were estimated using the difference in weekly hours worked between individuals with and without nocturia and average hourly earnings in the U.S. employed population from the U.S. Bureau of Labor Statistics. ${ }^{26}$ An estimate of the difference in weekly hours worked between cohorts was obtained from our NHANES analyses. Average hourly earnings were calculated using age-specific median hourly earnings reported by the Bureau of Labor Statistics' Current Population Survey, ${ }^{26}$ weighted by the proportion of the U.S. population within each age group. ${ }^{28}$ Indirect productivity costs associated with nocturia at a population level were calculated from individual level productivity costs and the estimated number of individuals with nocturia in the U.S. population. All unit costs extracted from the literature were adjusted for inflation and expressed in 2017 U.S. dollars using the medical services component of the Consumer Price Index.

\section{Statistical Analysis}

All analyses were performed by following NHANES analytic guidelines, ${ }^{30}$ and appropriate weights accounting for oversampling and survey nonresponse were used in all estimations to allow the results to be representative of the U.S. civilian noninstitutionalized population. Where appropriate, confidence intervals (CIs) were calculated using Taylor Series Linearization to account for the NHANES complex sampling design.

Individual characteristics were summarized using descriptive statistics. Outcomes were compared between cohorts overall and stratified by age group (i.e., 20-44 years, 45-64 years, and $65+$ years) using multivariable regressions adjusting for age, race, sex, body mass index, insurance status, education level, alcohol use, smoking status, and self-reported comorbid 
The Economic Burden of Nocturia on the U.S. Health Care System and Society:

A National Health and Nutrition Examination Survey Analysis

\section{FIGURE 1 Sample Selection}

\begin{tabular}{|c|c|c|c|}
\hline \multicolumn{4}{|c|}{$\begin{array}{l}\text { Individuals were enrolled in the NHANES study between survey cycles } 2005-2006 \text { and } 2013-2014 \\
\qquad N=50,965(100.0 \%)\end{array}$} \\
\hline \multicolumn{3}{|c|}{$\begin{array}{l}\text { Individuals were interviewed at home and went through the examination process } \\
\qquad \mathrm{n}=46,116(96.4 \%)\end{array}$} & \\
\hline \multicolumn{3}{|c|}{$\begin{array}{l}\text { Individuals were aged at least } 18 \text { years at the time of the interview } \\
\qquad n=29,228(59.5 \%)\end{array}$} & \\
\hline \multicolumn{3}{|c|}{$\begin{array}{l}\text { Individuals answered the NHANES questionnaires on kidney conditions and urology, } \\
\text { hospital utilization and access to care, medical conditions, and occupation } \\
\qquad n=22,737(77.8 \%)\end{array}$} & \\
\hline \multicolumn{3}{|c|}{$\begin{array}{l}\text { Individuals reported not being pregnant at the time of the interview } \\
\qquad n=22,300(98.1 \%)\end{array}$} & \\
\hline Study cohorts & Unweighted & Weighted $^{\mathrm{a}}$ & \\
\hline \multicolumn{3}{|c|}{ Main analysis-Nocturia defined as $\geq 2$ voids/night } & \\
\hline Individuals with nocturia & $6,782 \quad(30.4 \%)$ & $5,444 \quad(24.4 \%)$ & \\
\hline Individuals without nocturia & $15,518 \quad(69.6 \%)$ & $16,856 \quad(75.6 \%)$ & \\
\hline \multicolumn{3}{|c|}{ Main analysis-Nocturia defined as $\geq 2$ voids/night, excluding individuals with CHF and diabetes } & \\
\hline Individuals with nocturia & $5,155 \quad(27.0 \%)$ & $4,195 \quad(22.0 \%)$ & \\
\hline Individuals without nocturia & $13,952 \quad(73.0 \%)$ & $14,912 \quad(78.0 \%)$ & \\
\hline \multicolumn{3}{|c|}{ Sensitivity analysis-Nocturia defined as $\geq 1$ voids/night } & \\
\hline Individuals with nocturia & $14,901 \quad(66.8 \%)$ & $14,040 \quad(63.0 \%)$ & \\
\hline Individuals without nocturia & $7,399 \quad(33.2 \%)$ & $(37.0 \%)$ & \\
\hline
\end{tabular}

conditions (i.e., hypertension, stroke, diabetes, cancer, chronic obstructive pulmonary disease, coronary heart disease, congestive heart failure). HRU was compared using Poisson regressions and results were reported as adjusted IRRs. For work productivity, the number of weekly hours worked was compared using linear regressions, and results were reported as adjusted differences, while the odds of being employed were compared using logistic regression and results were reported as adjusted odds ratios (ORs). The odds of having a very good or excellent health status were compared using logistic regression and results were reported as adjusted ORs.

\section{Sensitivity Analyses}

Given the association between nocturia and certain conditions, including congestive heart failure and diabetes, where nocturia is regarded as a symptom of the primary disorder, ad hoc analyses were performed in which individuals with congestive heart failure or diabetes were excluded. ${ }^{18}$ However, due to data unavailability, it was not possible to account for the potential effect of sleep apnea and bladder storage problems, which may also be associated with nocturia.

To determine if the level of nocturia has an influence on its economic impact, a sensitivity analysis was conducted using a threshold of $\geq 1$ voids per night to define study cohorts and to identify nocturia prevalence.

\section{Results}

The NHANES study enrolled 50,965 participants between 2005-2006 and 2013-2014. The analytic sample included 22,300 individuals who met the study criteria (Figure 1). After 


\section{The Economic Burden of Nocturia on the U.S. Health Care System and Society: A National Health and Nutrition Examination Survey Analysis}

\section{TABLE 1 Individual Characteristics}

\begin{tabular}{|c|c|c|c|c|c|c|c|}
\hline $\begin{array}{l}\text { Individual } \\
\text { Characteristics }\end{array}$ & \begin{tabular}{|c|} 
Individuals with \\
Nocturia \\
$\mathrm{n}=5,444$
\end{tabular} & $\begin{array}{c}\text { Individuals } \\
\text { Without Nocturia } \\
\mathrm{n}=16,856\end{array}$ & $P$ Value ${ }^{a}$ & $\begin{array}{l}\text { Individual } \\
\text { Characteristics }\end{array}$ & $\begin{array}{c}\text { Individuals with } \\
\text { Nocturia } \\
\mathrm{n}=5,444\end{array}$ & \begin{tabular}{|c|} 
Individuals \\
Without Nocturia \\
$\mathrm{n}=16,856$
\end{tabular} & $P$ Value \\
\hline \multicolumn{4}{|l|}{ Demographics } & \multicolumn{4}{|l|}{ Comorbidities, n (\%) } \\
\hline Age, years, mean & 55.2 & 44.7 & $<0.001$ & & $2,670 \quad(49.0)$ & $4,498 \quad(26.7)$ & $<0.001$ \\
\hline Age, median (IQR $\left.{ }^{b}\right)$ & $55.2(43.0-68.1)$ & $43.2(30.5-55.6)$ & & Cancer & $856 \quad(15.7)$ & $1,322 \quad(7.8)$ & $<0.001$ \\
\hline $20-44, \mathrm{n}(\%)$ & $1,458 \quad(26.8)$ & $\begin{array}{ll}8,737 & (51.8) \\
5020 & (252)\end{array}$ & $<0.001$ & Prostate & $111 \quad(2.0)$ & $96 \quad(0.6)$ & $<0.001$ \\
\hline $\begin{array}{l}45-64, \mathrm{n}(\%) \\
65+, \mathrm{n}(\%)\end{array}$ & $\begin{array}{ll}2,224 & (40.9) \\
1,762 & (32.4)\end{array}$ & $\begin{array}{ll}5,929 & (35.2) \\
2,190 & (13.0)\end{array}$ & $<0.001$ & Diabetes & $898 \quad(16.5)$ & $1,095 \quad(6.5)$ & $<0.001$ \\
\hline Male, n (\%) & $2,355 \quad(43.3)$ & $8,653 \quad(51.3)$ & $<0.001$ & Sleep disorder & $701 \quad(12.9)$ & $1,249 \quad(7.4)$ & $<0.001$ \\
\hline \multicolumn{4}{|l|}{ Race, n (\%) } & \multirow{2}{*}{$\begin{array}{l}\text { Chronic obstructive } \\
\text { pulmonary disease }\end{array}$} & \multirow[t]{2}{*}{$613 \quad(11.3)$} & \multirow{2}{*}{$979 \quad(5.8)$} & \multirow{2}{*}{$<0.001$} \\
\hline White & $3,591 \quad(66.0)$ & $12,149 \quad(72.1)$ & $<0.001$ & & & & \\
\hline Black & $900 \quad(16.5)$ & $1,496 \quad(8.9)$ & $<0.001$ & Coronary heart disease & $(6.0)$ & $(2.4)$ & $<0.001$ \\
\hline Mexican American & $416 \quad(7.6)$ & $1,314 \quad(7.8)$ & 0.767 & Congestive heart failure & $(5.0)$ & (1.6) & $<0.001$ \\
\hline Other Hispanic & $272 \quad(5.0)$ & $(4.6)$ & 0.293 & Stroke & $(5.4)$ & $(2.0)$ & $<0.001$ \\
\hline Other race & $264 \quad(4.9)$ & 1,118 & $<0.001$ & \multirow{2}{*}{$\begin{array}{l}\text { Urinary leakage } \\
(>1 \text { monthly leakage })\end{array}$} & \multirow[t]{2}{*}{$1,651 \quad(30.3)$} & \multirow[t]{2}{*}{$2,309 \quad(13.7)$} & $<0.001$ \\
\hline Health insurance, n (\%) & $4,463 \quad(82.0)$ & $13,683 \quad(81.2)$ & 0.279 & & & & \\
\hline Private & $2,963 \quad(54.4)$ & $11,312 \quad(67.1)$ & $<0.001$ & Alcohol use ${ }^{c}, \mathrm{n}(\%)$ & $3,890 \quad(71.5)$ & $13,342 \quad(79.2)$ & $<0.001$ \\
\hline Medicare & $1,749 \quad(32.1)$ & $2,123 \quad(12.6)$ & $<0.001$ & Tobacco used, n (\%) & $2,815 \quad(51.7)$ & $7,480 \quad(44.4)$ & $<0.001$ \\
\hline Medicaid & $\begin{array}{ll}527 & (9.7) \\
\end{array}$ & $718 \quad(4.3)$ & $<0.001$ & Current smoker & $1,205 \quad(22.1)$ & $3,554 \quad(21.1)$ & 0.169 \\
\hline $\begin{array}{l}\text { Medigap } \\
\text { Other health insur }\end{array}$ & $\begin{aligned} 28 & (0.5) \\
327 & (50)\end{aligned}$ & $\begin{array}{r}51 \\
887\end{array}$ & $\begin{array}{l}0.029 \\
0126\end{array}$ & Former smoker & $1,610 \quad(29.6)$ & $3,926 \quad(23.3)$ & $<0.001$ \\
\hline $\begin{array}{l}\text { Other health insurance } \\
\text { Unknown health }\end{array}$ & $\begin{array}{rr}322 & (5.9) \\
6 & (0.1)\end{array}$ & $\begin{array}{l}(5.2) \\
(0.1)\end{array}$ & $\begin{array}{l}0.126 \\
0.616\end{array}$ & $\mathrm{BMI}^{\mathrm{e}}$, mean & 28.8 & 27.3 & $<0.001$ \\
\hline insurance & & & & BMI, median (IQRb) & $28.2(24.4-32.9)$ & $26.6(23.4-30.7)$ & \\
\hline High school, n (\%) & $4,081 \quad(75.0)$ & $14,502 \quad(86.0)$ & $<0.001$ & Underweight & (1.1) & $(1.5)$ & 0.046 \\
\hline Clinical characteristics & & & & & & & \\
\hline mber of vo & ght), n (\%) & & & Normal weight & $1,353 \quad(24.9)$ & $5,626 \quad(33.4)$ & $<0.001$ \\
\hline 1 & $0 \quad(0)$ & $8,596 \quad(51.0)$ & - & and $\leq 24.9), n(\%)$ & & & \\
\hline 2 & $3,439 \quad(63.2)$ & $(0)$ & - & Over & $1,722 \quad(31.6)$ & $5,751 \quad(34.1)$ & 0.010 \\
\hline 3 & $1,338 \quad(24.6)$ & $(0)$ & - & $(>24.9$ and $<30)$, n ( & & & \\
\hline 4 & $379 \quad(7.0)$ & $(0)$ & - & Obese ( $\geq 30), \mathrm{n}(\%)$ & $2,127 \quad(39.1)$ & $4,761 \quad(28.2)$ & $<0.001$ \\
\hline $5+$ & $288 \quad(5.3)$ & $(0)$ & - & Unknown BMI & $(3.4)$ & $(2.8)$ & 0.043 \\
\hline $\begin{array}{l}\text { aP values were measured u } \\
\text { bIQR corresponds to the fir } \\
\text { 'Alcohol use was defined a } \\
\text { dTobacco use included curr } \\
\text { smoking as of the interview } \\
\text { reported not smoking as of } \\
{ }^{e} B M I \text { was defined as the in }\end{array}$ & $\begin{array}{l}\text { ind former smoke } \\
\text { her occasionally o } \\
\text { nterview. } \\
\text { lual's weight in kil }\end{array}$ & $\begin{array}{l}\text { a) current smoke } \\
\text { ery day); (b) for } \\
\text { ams divided by th }\end{array}$ & $\begin{array}{l}\text { re defi } \\
\text { moker } \\
\text { are of }\end{array}$ & $\begin{array}{l}\text { individuals who smoked a } \\
\text { lefined as individuals whe } \\
\text { eight in meters }\left(\mathrm{kg} / \mathrm{m}^{2}\right) \text {. }\end{array}$ & $\begin{array}{l}t 100 \text { cigarettes } \\
\text { ked at least } 100\end{array}$ & $\begin{array}{l}\text { ir lifetime and } \\
\text { ettes in their li }\end{array}$ & $\begin{array}{l}\text { reported } \\
\text { but who }\end{array}$ \\
\hline
\end{tabular}

the application of sampling weights, $24.4 \%$ of the sample was classified as having nocturia (i.e., $\geq 2$ voids/night) and $75.6 \%$ without nocturia (i.e., <2 voids/night; Figure 1), consistent with previously published epidemiologic NHANES analyses. ${ }^{2}$ Compared with individuals without nocturia, those with nocturia were older (mean age: 55.2 vs. 44.7 years, respectively; $P<0.001)$ and less likely to be male $(43.3 \%$ and $51.3 \%$, respectively; $P<0.001$ ). Among individuals with nocturia, $63.2 \%$ reported 2 voids, $24.6 \%$ reported 3 voids, $7.0 \%$ reported 4 voids, and $5.3 \%$ reported $\geq 5$ voids per night. The most common comorbid condition was hypertension, with a statistically significantly higher proportion in individuals with nocturia than without ( $49.0 \%$ vs. $26.7 \%$, respectively; $P<0.001$; Table 1 ).

\section{HRU, Work Productivity, and Health Status}

Compared with individuals without nocturia, those with nocturia had statistically significantly higher HRU, including 60\% higher hospitalization rates (IRR $=1.60 ; P<0.001)$ and $19 \%$ higher rates of outpatient visits (IRR $=1.19 ; P<0.001$; Table 2). These comparisons remained statistically significant across age groups, with the difference being more marked among individuals aged 20-44 years (hospitalizations: IRR $=1.79$; outpatient visits: IRR $=1.24$; both $P<0.001$ ). Individuals with nocturia exhibited statistically significantly reduced work productivity compared with those without nocturia, including fewer hours worked per week (difference $=-3.91 ; P<0.001$ ) and lower odds of being employed $(\mathrm{OR}=0.66 ; P<0.001$; Table 2$)$. Individuals 


\section{The Economic Burden of Nocturia on the U.S. Health Care System and Society: A National Health and Nutrition Examination Survey Analysis}

TABLE 2 HRU, Work Productivity, and Health Status Stratified by Age

\begin{tabular}{|c|c|c|c|c|}
\hline \multirow{3}{*}{$\begin{array}{l}\text { Study Outcome } \\
\text { HRU }\end{array}$} & \multicolumn{4}{|c|}{ Comparison of Individuals with vs. Without Nocturia } \\
\hline & \multicolumn{2}{|c|}{$\begin{array}{l}\text { Total Sample } \\
(\mathrm{N}=22,300)\end{array}$} & \multicolumn{2}{|c|}{$\begin{array}{l}\text { Excluding Individuals with CHF/Diabetes } \\
\qquad(\mathrm{n}=19,107)\end{array}$} \\
\hline & $\operatorname{IRR}(95 \% \mathrm{CI})^{\mathrm{a}}$ & \multirow{2}{*}{$\begin{array}{c}P \text { Value } \\
<0.001\end{array}$} & IRR $(95 \% \mathrm{CI})^{\mathrm{a}}$ & \multirow{2}{*}{$\begin{array}{c}P \text { Value } \\
<0.001\end{array}$} \\
\hline Hospitalization & $1.60 \quad(1.39,1.84)$ & & $1.69(1.44,1.99)$ & \\
\hline Aged $20-44$ years & $1.79 \quad(1.36,2.35)$ & $<0.001$ & $1.83(1.38,2.42)$ & $<0.001$ \\
\hline Aged $45-64$ years & $1.57 \quad(1.30,1.88)$ & $<0.001$ & $1.55 \quad(1.22,1.97)$ & $<0.001$ \\
\hline Aged $65+$ years & $1.30 \quad(1.10,1.53)$ & 0.003 & $1.41 \quad(1.15,1.73)$ & 0.001 \\
\hline Outpatient visits & $1.19 \quad(1.15,1.23)$ & $<0.001$ & $1.22 \quad(1.17,1.27)$ & $<0.001$ \\
\hline Aged 20-44 years & $1.24 \quad(1.15,1.34)$ & $<0.001$ & $1.27 \quad(1.17,1.38)$ & $<0.001$ \\
\hline Aged $45-64$ years & $1.16 \quad(1.09,1.23)$ & $<0.001$ & $1.18 \quad(1.10,1.26)$ & $<0.001$ \\
\hline Aged $65+$ years & $1.12 \quad(1.07,1.17)$ & $<0.001$ & $1.16 \quad(1.08,1.23)$ & $<0.001$ \\
\hline Work Productivity & Difference $(95 \% \text { CI })^{b}$ & $P$ Value & Difference $(95 \% \text { CI })^{b}$ & $P$ Value \\
\hline Weekly hours worked & $-3.91(-4.75,-3.07)$ & $<0.001$ & $-3.88(-4.77,-2.99)$ & $<0.001$ \\
\hline Aged $20-44$ years & $-2.69(-4.36,-1.02)$ & 0.002 & $-2.68(-4.41,-0.95)$ & 0.003 \\
\hline Aged 45-64 years & $-4.13(-5.50,-2.76)$ & $<0.001$ & $-3.97(-5.42,-2.52)$ & $<0.001$ \\
\hline \multirow[t]{2}{*}{ Aged $65+$ years } & $-2.19(-3.17,-1.21)$ & $<0.001$ & $-1.94(-2.98,-0.89)$ & $<0.001$ \\
\hline & OR $(95 \% \mathrm{CI})^{\mathrm{c}}$ & $P$ Value & OR $(95 \% \mathrm{CI})^{\mathrm{c}}$ & $P$ Value \\
\hline Employment status & $0.66 \quad(0.60,0.72)$ & $<0.001$ & $0.65 \quad(0.60,0.72)$ & $<0.001$ \\
\hline Aged 20-44 years & $0.70 \quad(0.60,0.82)$ & $<0.001$ & $0.70 \quad(0.60,0.83)$ & $<0.001$ \\
\hline Aged 45-64 years & $0.65 \quad(0.56,0.75)$ & $<0.001$ & $0.64 \quad(0.55,0.75)$ & $<0.001$ \\
\hline Aged $65+$ years & $0.64 \quad(0.51,0.79)$ & $<0.001$ & $0.67 \quad(0.52,0.87)$ & 0.003 \\
\hline Current Health Status & OR $(95 \% \mathrm{CI})^{\mathrm{d}}$ & $P$ Value & OR $(95 \% \mathrm{CI})^{\mathrm{d}}$ & $P$ Value \\
\hline Very good or excellent & $0.54 \quad(0.48,0.60)$ & $<0.001$ & $0.52 \quad(0.46,0.58)$ & $<0.001$ \\
\hline Aged 20-44 years & $0.63 \quad(0.53,0.76)$ & $<0.001$ & $0.62 \quad(0.52,0.75)$ & $<0.001$ \\
\hline Aged 45-64 years & $0.43 \quad(0.35,0.53)$ & $<0.001$ & $0.42 \quad(0.34,0.52)$ & $<0.001$ \\
\hline Aged $65+$ years & $0.66 \quad(0.55,0.79)$ & $<0.001$ & $0.60 \quad(0.48,0.75)$ & $<0.001$ \\
\hline \multicolumn{5}{|c|}{$\begin{array}{l}\text { aAn IRR }>1 \text { indicates that individuals with nocturia had more hospitalizations or outpatient visits compared with individuals without nocturia. } \\
{ }^{b} \text { A difference }<0 \text { indicates that individuals with nocturia worked fewer hours weekly compared with individuals without nocturia. } \\
{ }^{c} \text { An odds ratio }<1 \text { indicates that individuals with nocturia are less likely to report being employed compared with individuals without nocturia. } \\
{ }^{A} \text { An odds ratio < } 1 \text { indicates that individuals with nocturia are less likely to report being in very good or excellent health compared with individuals without nocturia. } \\
C H F=\text { congestive heart failure; } C I=\text { confidence interval; HRU = health care resource use; IRR=incidence rate ratio; OR=odds ratio. }\end{array}$} \\
\hline
\end{tabular}

with nocturia were $46 \%$ less likely to report being in very good or excellent health $(\mathrm{OR}=0.54 ; \mathrm{P}<0.001)$, a trend that was observed in all age groups (20-44 years: $\mathrm{OR}=0.63 ; 45-64$ years: $\mathrm{OR}=0.43 ; 65+$ years: $\mathrm{OR}=0.66$; all $P<0.001$; Table 2). In the subset of individuals who did not report congestive heart failure or diabetes, results were largely similar to those of the overall sample. In the sensitivity analyses using a threshold of $\geq 1$ voids per night to define study cohorts, nocturia was associated with higher HRU, lower work productivity, and poorer self-reported health overall, but not consistently across age groups, notably the 65+ year old age group (Appendix A, available in online article).

\section{Economic Burden of Nocturia}

The prevalence of nocturia in the U.S. population was estimated to be $14.4 \%$ in individuals aged $20-44$ years (i.e., $15,630,493$ individuals), $27.1 \%$ in those aged $45-64$ years (i.e., $22,842,908$ individuals), and $45.2 \%$ in those aged $65+$ years (i.e., 22,983,833 individuals) based on estimates reported in
Vaughan et al. for $\geq 2$ voids per night and the U.S. population in 2017. 2,28 Using these values, total excess direct health care costs were estimated at $\$ 62.9$ billion, including $\$ 47.6$ and $\$ 15.3$ billion in excess hospitalization and outpatient costs, respectively. Total excess indirect productivity costs were estimated at $\$ 151.7$ billion. Altogether, total excess direct health care and indirect productivity costs were estimated at $\$ 214.5$ billion (range $=\$ 94.0-\$ 231.1$ billion, based on different prevalence estimates for $\geq 2$ voids per night), equivalent to $\$ 3,491$ per individual with nocturia (Table 3). Indirect productivity costs were the main driver of the total costs associated with nocturia (70.7\%). Total excess costs per individual varied by age group and were highest for individuals aged $45-64$ years ( $\$ 4,525$ vs. $\$ 3,231$ in those aged 20-44 years and $\$ 2,639$ in those aged $65+$ years; Table 4). Accordingly, $48.2 \%$ of total excess costs were incurred by individuals aged 45-64 years, compared with $23.5 \%$ and $28.3 \%$ for individuals aged $20-44$ and $65+$ years, respectively. 
TABLE 3 Summary of Excess Costs Associated with Nocturia in the United States in 2017

\begin{tabular}{|c|c|c|c|}
\hline Component & $\begin{array}{l}\text { Excess Costs }^{\mathrm{a}} \\
\text { (2017 USD) }\end{array}$ & $\begin{array}{c}\text { Minimum Prevalence }{ }^{b} \\
\text { (2017 USD) }\end{array}$ & $\begin{array}{c}\text { Maximum Prevalencec } \\
\text { (2017 USD) }\end{array}$ \\
\hline Total excess direct health care costs & $62,863,560,007$ & $28,876,072,235$ & $67,768,043,702$ \\
\hline Excess hospitalization costs & $47,591,010,669$ & $21,864,595,980$ & $51,287,348,211$ \\
\hline Aged $20-44$ years & $14,221,168,129$ & $3,702,428,339$ & $13,024,141,644$ \\
\hline Aged $45-64$ years & $15,768,493,293$ & $5,871,620,891$ & $17,045,890,200$ \\
\hline Aged $65+$ years & $17,601,349,247$ & $12,290,546,750$ & $21,217,316,366$ \\
\hline Excess outpatient costs & $15,272,549,338$ & $7,011,476,255$ & $16,480,695,491$ \\
\hline Aged $20-44$ years & $4,368,432,570$ & $1,137,305,206$ & $4,000,732,151$ \\
\hline Aged $45-64$ years & $5,338,544,741$ & $1,987,882,434$ & $5,771,017,293$ \\
\hline Aged $65+$ years & $5,565,572,027$ & $3,886,288,615$ & $6,708,946,047$ \\
\hline Total excess indirect productivity costs & $151,667,800,937$ & $65,115,651,454$ & $163,345,671,307$ \\
\hline Aged $20-44$ years & $31,918,442,222$ & $8,309,847,965$ & $29,231,797,895$ \\
\hline Aged 45-64 years & $82,268,205,819$ & $30,633,726,825$ & $88,932,707,606$ \\
\hline Aged $65+$ years & $37,481,152,895$ & $26,172,076,665$ & $45,181,165,806$ \\
\hline Total excess direct health care and indirect costs & $214,531,360,944$ & $93,991,723,689$ & $231,113,715,009$ \\
\hline Total excess direct health care and indirect costs per individual with nocturia ${ }^{\mathrm{d}}$ & 3,491 & 3,284 & 3,464 \\
\hline \multicolumn{4}{|c|}{$\begin{array}{l}\text { aThe prevalence of nocturia in the population was calculated using sex-specific prevalence reported in Vaughan et al. (2016), }{ }^{2} \text { weighted using the sex distribution of the } \\
\text { U.S. population. Using this prevalence, the number of individuals with } \geq 2 \text { voids per night in the United States in } 2017 \text { was estimated to be 15,630,493 for those aged } \\
20-44 \text { years; } 22,842,908 \text { for those aged } 45-64 \text { years; and 22,983,833 for those aged } 65+\text { years. } \\
\text { bThe prevalence of nocturia in the population was calculated using the minimum sex-specific prevalence reported in Bosch et al. (2010), } 29 \text { weighted using the sex distribu- } \\
\text { tion of the U.S. population. Using this prevalence, the number of individuals with } \geq 2 \text { voids per night in the United States in } 2017 \text { was estimated to be 4,069,341 for those } \\
\text { aged 20-44 years; 8,505,879 for those aged 45-64 years; and 16,048,990 for those aged } 65+\text { years. } \\
\text { cThe prevalence of nocturia in the population was calculated using the maximum sex-specific prevalence reported in Bosch et al., } 29 \text { weighted using the sex distribution of } \\
\text { the U.S. population. Using this prevalence, the number of individuals with } \geq 2 \text { voids per night in the United States in } 2017 \text { was estimated to be 14,314,840 for those aged } \\
20-44 \text { years; } 24,693,399 \text { for those aged } 45-64 \text { years; and 27,705,562 for those aged } 65+\text { years. } \\
\text { dTotal excess costs per individual were estimated by dividing the total excess costs by the estimated number of individuals with nocturia in the United States. In sensitivity } \\
\text { analyses, the minimum and maximum prevalence for each age and gender group were used, such that the overall distribution of individuals per age and gender for these } \\
\text { analyses may be different from those in the main analysis. Accordingly, costs per individual obtained in the main analyses may not be included within those obtained using } \\
\text { minimum and maximum prevalence estimates. }\end{array}$} \\
\hline
\end{tabular}

In the sensitivity analyses using $\geq 1$ voids per night to define study cohorts and to identify nocturia prevalence, total excess direct health care and indirect costs associated with nocturia were estimated at $\$ 167.3$ billion (range $=\$ 127.5-\$ 207.0$ billion, based on different prevalence estimates for $\geq 1$ voids per night), comprising $\$ 25.5$ billion in direct health care costs and $\$ 141.8$ billion in indirect excess productivity costs, equivalent to $\$ 1,452$ per individual with nocturia (see Appendix B, available in online article).

\section{Discussion}

In this large, nationally representative sample of U.S. adults, nocturia was associated with substantial HRU, work productivity loss, and poorer self-reported health when evaluated both at the clinically relevant threshold of $\geq 2$ voids per night and at $\geq 1$ voids per night in a sensitivity analysis. More specifically, compared with individuals without nocturia, those with nocturia ( $\geq 2$ voids/night) had $60 \%$ higher rates of hospitalization, $19 \%$ higher rates of outpatient visits, and were $46 \%$ less likely to report being in very good or excellent health. Furthermore, individuals with nocturia were less likely to be employed and worked fewer hours per week. The total excess direct health care and indirect costs were estimated at $\$ 214.5$ billion, equivalent to $\$ 3,491$ per individual with nocturia, with direct costs to the health care system accounting for $29 \%$ of total costs.

Few previous studies have examined the economic burden of nocturia. These studies mostly focused on individual components of costs (i.e., direct or indirect costs only or costs related to falls/fractures), with no estimate of overall costs associated with nocturia, or were based on demographically or geographically restricted populations (e.g., older adults, individuals in a particular U.S. region, or individuals outside the United States). ${ }^{15,19-22}$

A recent European study by Weidlich et al. (2017) estimated the total excess direct and indirect costs associated with nocturia at a population level. ${ }^{22}$ Based on a physician survey, authors estimated that patients had on average 3.6 medical visits related to nocturia, and $2.6 \%$ of patients would have a hospitalization due to nocturia annually. Overall, the estimated annual direct costs of HRU attributable to managing nocturia ranged from $€ 185$ to $€ 463$ (i.e., $\approx \$ 208$-\$520) per individual with nocturia, depending on the country. ${ }^{22}$ Differences in the health care systems in Europe versus the United States and study methodologies may explain lower estimates compared with the present study. For example, unlike the present study, Weidlich et al. 


\section{The Economic Burden of Nocturia on the U.S. Health Care System and Society:}

A National Health and Nutrition Examination Survey Analysis

\section{TABLE 4 Estimated Annual Costs Associated with Nocturia per Individual}

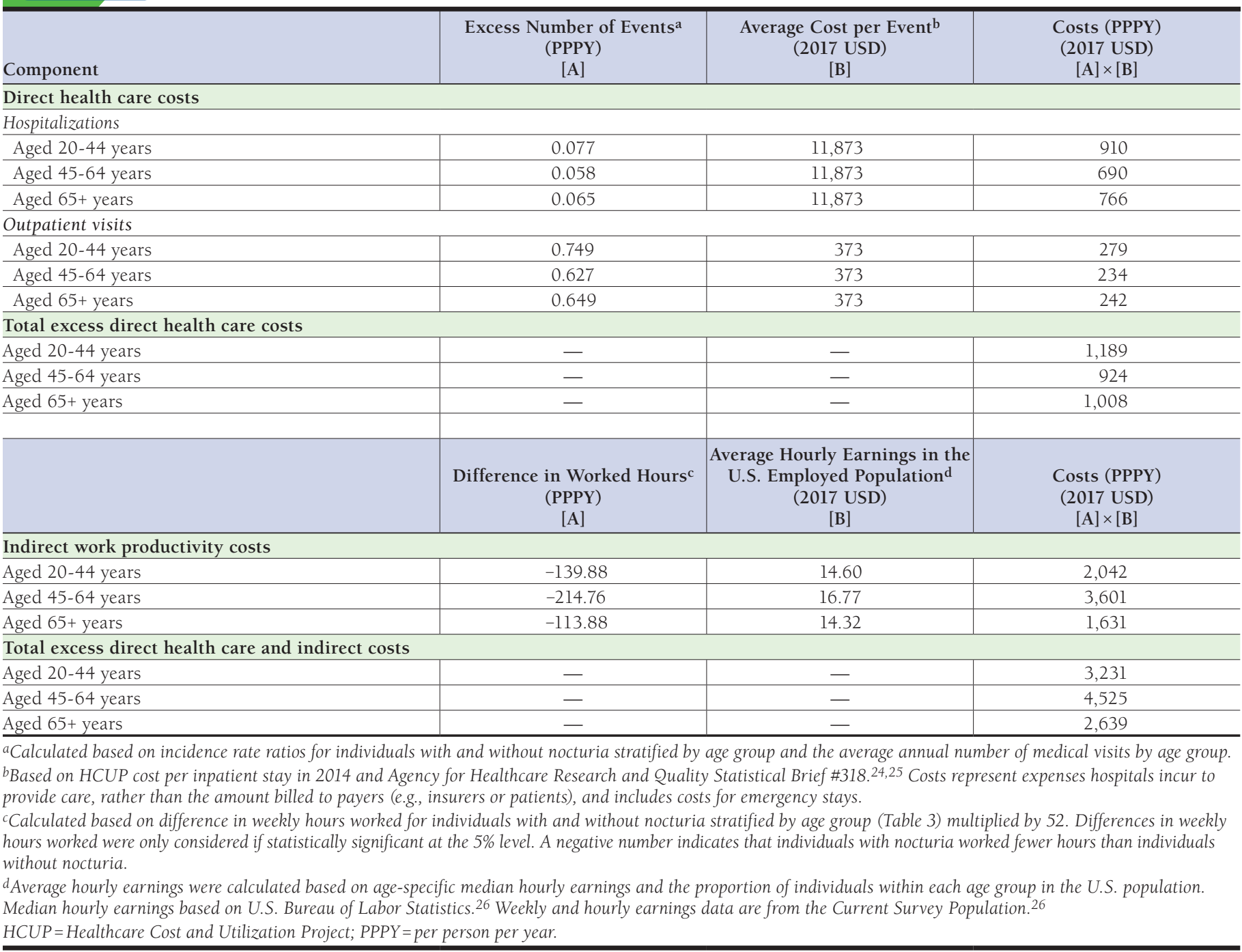

assessed the costs of managing nocturia, but not those associated with a potential deterioration of health beyond nocturia symptoms. In addition to health care costs, they evaluated the annual work productivity loss due to nocturia at 1.1 months or $€ 655-€ 1,781$ (i.e., $\approx \$ 735-\$ 1,999$ ) per individual depending on the country, consistent with our findings. Overall, authors found that indirect productivity costs were the main driver of the economic burden of nocturia, as per the present study. ${ }^{22}$

Hafner et al. (2019) also surveyed employed individuals and reported indirect productivity costs across the United Kingdom, Australia, Malaysia, Hong Kong, Thailand, Singapore, and Sri Lanka. ${ }^{20}$ Absenteeism and reduced work productivity were associated with an annual productivity loss of 7.6 days (i.e., 60.1 hours assuming 8 hours worked/day) among employed individuals, which represents about half of the work loss found in the present study. However, this is expected, because the study did not capture work productivity loss due to unemployment as done in the present study. Indeed, our results show that individuals with nocturia are 30\%-35\% less likely to be employed than those without nocturia.

A recent U.S. study of 899 residents of the greater Los Angeles area found that, among individuals diagnosed with nocturia, those with $\geq 2$ voids per night had significantly higher health care costs compared with individuals with 1 void per night ( $\$ 1,144$ vs. $\$ 798$ per patient per month) and incurred an annual work loss of 26.4 days (i.e., 211.2 hours assuming 8 hours worked/day). ${ }^{15}$ Accordingly, the estimated HRU and productivity costs associated with $\geq 2$ voids per night among individuals diagnosed with nocturia in that study are larger than those estimated among individual self-reporting $\geq 2$ voids 


\section{The Economic Burden of Nocturia on the U.S. Health Care System and Society: A National Health and Nutrition Examination Survey Analysis}

per night in the present study. It is possible that the effect of nocturia is more severe among individuals seeking care for their condition than in the overall population experiencing nocturia symptoms. Other differences in sample characteristics may also explain differences with the present study. For example, individuals in the present study were younger on average. Finally, it should be noted that the study did not evaluate the overall economic burden of nocturia in the United States.

A single study previously estimated indirect productivity costs associated with nocturia in the United States based on a U.S. sample. The authors found that nocturia was associated with an annual loss of 115 hours of work for women and 142 hours for men, which translated into indirect productivity costs of $\$ 61$ billion in 2008 (i.e., $\$ 69.5$ billion in 2017 USD). ${ }^{19}$ Although this figure is lower than our calculations using prevalence values reported by Vaughan et al. (i.e., $\$ 151.7$ billion), it is similar to that using the minimum prevalence reported in the literature ( $\$ 65.1$ billion).

A key differentiator and strength of our analyses is that we examined the effect of nocturia on overall health, HRU, and costs according to age, whereas none of the recent economic burden studies conducted age group-specific analyses. .5,19,20,22 $^{2}$ Among the few studies that reported the effect of nocturia on younger adults, another NHANES study found that nocturia was a strong predictor of mortality among those aged younger than 65 years. ${ }^{8}$ Other studies indicated that younger individuals may experience more sleep disruption and related problems than older individuals. ${ }^{31}$ Disrupted sleep, regardless of cause, can have a considerable effect on the overall health and wellbeing of individuals, which can be particularly detrimental to younger individuals with more active and demanding working lives. $^{32}$ In the present study, nearly one quarter of the total excess costs associated with nocturia was borne by individuals aged younger than 45 years, and this proportion reached nearly one third when considering only health care costs. Yet, nocturia is often overlooked in younger populations, and our results highlight the need for greater awareness about this condition across all age groups.

The present study found that nocturia is associated with a large economic burden, despite the fact that it has been relatively understudied compared with other conditions. In comparison, the annual economic burden associated with overactive bladder (another LUTS) in the United States is estimated to reach \$34-\$89 billion in 2017 USD, less than half that of nocturia. ${ }^{33,34}$ Although costs associated with nocturia remain relatively low at the individual level (i.e., $\$ 3,491$ per individual with nocturia), its large prevalence implies an economic burden comparable to other conditions. Our findings suggest that the economic burden associated with nocturia in the United States would be almost as high as that of major depressive disorder (i.e., $\$ 241$ billion) and approximately two thirds of that of diabetes (i.e., $\$ 327$ billion). ${ }^{35,36}$
It is increasingly recognized that nocturia is a highly prevalent, chronic condition that merits greater awareness, and warrants full consideration as a clinically significant target for therapeutic intervention due to its wide ranging and serious repercussions. Given the substantial societal economic impact of nocturia, all stakeholders, including patients, clinicians, and policy decision makers should be alerted to the importance of proactive and timely identification and treatment of nocturia to reduce the burden on individuals and society. Although nocturia should initially be managed by optimizing the treatment of underlying metabolic conditions, including diabetes, congestive heart failure, and sleep apnea, antidiuretic pharmacologic intervention should be considered following the failure of conservative (behavioral) and metabolic corrections. ${ }^{37}$ Up to $88 \%$ of patients with nocturia have nocturnal polyuria, where nocturnal urine output is increased..$^{38}$ In these patients, treatment with desmopressin, a structural analogue of the natural antidiuretic hormone vasopressin, can reduce frequency of nocturnal voiding. ${ }^{39}$ The lack of disease awareness and available treatment options combined with patients' reluctance to discuss symptoms often leads to suboptimal management of nocturia, resulting in substantial health-related and economic burden to the U.S. health care system and society.

\section{Limitations}

Our study has some limitations to consider. The study cohorts were defined based on the number of voids rather than other clinically relevant outcomes, such as bother of voiding or hours of undisturbed sleep, which are not reported in the NHANES database. Outcomes were self-reported and may be prone to recall bias. Studies suggest that self-reported HRU is typically underreported, especially for individuals with substantial HRU ${ }^{40}$ Additionally, because the NHANES database does not provide information on HRU costs or details on the nature of hospitalizations and outpatient visits, associated health care costs were estimated based on aggregated average costs in the U.S. population, which may not be representative of the average costs incurred among patients with nocturia. Likewise, since aggregated costs were used, no information on out-ofpocket costs was available. Further, data on pharmacy costs, presenteeism, and unpaid work/leisure time were not available in the NHANES database, which may result in underestimation of direct and indirect costs.

The NHANES sample is based on a noninstitutionalized community-dwelling adult population and may not be entirely representative of the overall population of adults with nocturia (e.g., individuals in long-term care facilities). Moreover, our analysis included "all-cause" nocturia because it was not possible to determine the specific cause of nocturia. However, associations remained robust when excluding patients with congestive heart failure and diabetes, suggesting that other common causes of nocturia, including nocturnal polyuria or LUTS, may also be important cost drivers. 


\section{The Economic Burden of Nocturia on the U.S. Health Care System and Society: A National Health and Nutrition Examination Survey Analysis}

Finally, although multivariable adjustment was carried out, residual confounding due to unobserved characteristics may remain.

\section{Conclusions}

Nocturia is associated with increased HRU, lower work productivity, and poorer self-reported health. The estimated annual economic burden associated with nocturia was $\$ 214.5$ billion. Accordingly, nocturia imposes a substantial economic burden on the U.S. health care system and society. Improved awareness of the societal effect of nocturia, timely evaluation, and effective management may result in reduced resource utilization while improving disease-associated burden to patients.

\section{Authors}

JAY JHAVERI, MD, MPH, Ferring Pharmaceuticals, Parsippany, New Jersey. MARJOLAINE GAUTHIER-LOISELLE, PhD, and PATRICK GAGNON-SANSCHAGRIN, MSc, Analysis Group, Montreal, Quebec, Canada. ERIC Q. WU, PhD, Analysis Group, Boston, Massachusetts.

AUTHOR CORRESPONDENCE: Marjolaine Gauthier-Loiselle, PhD, Analysis Group, 1190 avenue des Canadiens-de-Montréal, Tour Deloitte, Ste. 1500, Montréal, 2C H3B 0M7. Tel.: 514.394.4486; E-mail: Marjolaine.Gauthier-Loiselle@analysisgroup.com.

\section{DISCLOSURES}

This work was supported by Ferring Pharmaceuticals, which contributed to and approved the study design and participated in the interpretation of data, review, and approval of the manuscript. Gauthier-Loiselle, GagnonSanschagrin, and $\mathrm{Wu}$ are employees of Analysis Group, which received consultancy fees from Ferring Pharmaceuticals for work on this study. Jhaveri is a full-time employee of Ferring Pharmaceuticals.

Parts of this work were presented as a poster presentation at AMCP Nexus 2018; October 22-25, 2018; Orlando, FL.

\section{ACKNOWLEDGMENTS}

The authors thank Amy Guo, PhD, of Ferring Pharmaceuticals, for her important contribution to this work, and Elizabeth Serra, MPH, of Analysis Group, for analytical support. Medical writing assistance was provided by Sara Kaffashian, PhD, of Analysis Group.

\section{REFERENCES}

1. Fine ND, Weiss JP, Wein AJ. Nocturia: consequences, classification, and management. F1000Res. 2017;6:1627.

2. Vaughan CP, Fung CH, Huang AJ, Johnson TMN, Markland AD. Differences in the association of nocturia and functional outcomes of sleep by age and gender: a cross-sectional, population-based study. Clin Ther. 2016;38(11):2386-93 e2381

3. Miotla P, Dobruch J, Lipinski M, et al. Diagnostic and therapeutic recommendations for patients with nocturia. Cent European J Urol. 2017;70(4):388-93.

4. Bosch JL, Weiss JP. The prevalence and causes of nocturia. J Urol. 2013;189(1 Suppl):S86-92
5. Tikkinen KA, Auvinen A, Johnson TM 2nd, et al. A systematic evaluation of factors associated with nocturia - the population-based FINNO study. Am J Epidemiol. 2009;170(3):361-68.

6. Fitzgerald MP, Litman HJ, Link CL, McKinlay JB. The association of nocturia with cardiac disease, diabetes, body mass index, age and diuretic use: results from the BACH survey. J Urol. 2007;177(4):1385-89.

7. van der Vaart CH, Roovers JP, de Leeuw JR, Heintz AP. Association between urogenital symptoms and depression in community-dwelling women aged 20 to 70 years. Urology. 2007;69(4):691-96.

8. Kupelian V, Fitzgerald MP, Kaplan SA, Norgaard JP, Chiu GR, Rosen RC. Association of nocturia and mortality: results from the Third National Health and Nutrition Examination Survey. J Urol. 2011;185(2):571-77.

9. Asplund R, Henriksson S, Johansson S, Isacsson G. Nocturia and depression. BJU Int. 2004;93(9):1253-56.

10. Kupelian V, Wei JT, O'Leary MP, Norgaard JP, Rosen RC, McKinlay JB. Nocturia and quality of life: results from the Boston area community health survey. Eur Urol. 2012;61(1):78-84.

11. Van Dijk MM, Wijkstra H, Debruyne FM, De La Rosette JJ, Michel MC. The role of nocturia in the quality of life of men with lower urinary tract symptoms. BJU Int. 2010;105(8):1141-46.

12. Chung E. Desmopressin and nocturnal voiding dysfunction: clinical evidence and safety profile in the treatment of nocturia. Expert Opin Pharmacother. 2018;19(3):291-98.

13. Kujubu DA, Aboseif SR. An overview of nocturia and the syndrome of nocturnal polyuria in the elderly. Nat Clin Pract Nephrol. 2008;4(8):426-35.

14. Kobelt G, Borgstrom F, Mattiasson A. Productivity, vitality and utility in a group of healthy professionally active individuals with nocturia. BJU Int. 2003;91(3):190-95.

15. Dmochowski R, Brucker BM, Cole E, et al. Economic burden of illness in adult patients with nocturia. J Manag Care Spec Pharm. 2019;25(5):593-604. Available at: https://www.jmcp.org/doi/10.18553/jmcp.2019.18067.

16. Holm Larsen T, Weiss J, Langkilde LK. Economic burden of nocturia in the US adult population. J Urol. 2010;183(Suppl 4):el.

17. MacDiarmid S, Rosenberg M. Overactive bladder in women: symptom impact and treatment expectations. Curr Med Opin Res. 2005;21(9):1413-21.

18. Weiss JP. Nocturia: focus on etiology and consequences. Rev Urol. 2012;14(3-4):48-55.

19. Holm-Larsen T. The economic impact of nocturia. Neurourol Urodyn. 2014;33(Suppl 1):S10-14.

20. Hafner M, Pollard J, Troxel WM, et al. How frequent night-time bathroom visits can negatively impact sleep, well-being and productivity: examining the associations between nocturia, well-being and economic outcomes in a working-age population. 2019. Available at: https://www.rand.org/pubs/ research_reports/RR3043.html. Accessed August 28, 2019.

21. Carroll NV, Slattum PW, Cox FM. The cost of falls among the communitydwelling elderly. J Manag Care Pharm. 2005;11(4):307-16. Available at: https://www.jmcp.org/doi/10.18553/jmcp.2005.11.4.307.

22. Weidlich D, Andersson FL, Oelke M, Drake MJ, Jonasson AF, Guest JF. Annual direct and indirect costs attributable to nocturia in Germany, Sweden, and the UK. Eur J Health Econ. 2017;18(6):761-71.

23. Centers for Disease Control and Prevention, National Center for Health Statistics. National Health and Nutrition Examination Survey: NCHS Research Ethics Review Board (ERB) approval. Last reviewed November 29, 2017. Available at: https://www.cdc.gov/nchs/nhanes/irba98.htm. Accessed August 28, 2019.

24. Agency for Healthcare Research and Quality. Statistical brief \#318: expenses and characteristics of physician visits in different ambulatory care settings, 2008. March 2011. Available at: https://meps.ahrq.gov/data_files/ publications/st318/stat318.shtml. Accessed August 282019. 
25. Healthcare Cost acnd Utilization Project. H-CUP fast stats: trends in inpatient stays. Available at: https://www.hcup-us.ahrq.gov/faststats/ NationalTrendsServlet. Accessed August 28, 2019.

26. U.S. Department of Labor, Bureau of Labor Statistics. Labor force statistics from the current population survey. 2018. Available at: https:/www.bls gov/cps/. Accessed August 282019.

27. Hashim H, Blanker MH, Drake MJ, et al. International Continence Society (ICS) report on the terminology for nocturia and nocturnal lower urinary tract function. Neurourol Urodyn. 2019;38(2):499-508.

28. U.S. Census Bureau. Annual estimates of the resident population for selected age groups by sex for the United States, states, counties, and Puerto Rico commonwealth and municipios: April 1, 2010, to July 1, 2017. 2018. Available at: https://factfinder.census.gov/faces/tableservices/jsf/pages/productview.xhtml?src=blkmk. Accessed August 28, 2019.

29. Bosch JL, Weiss JP. The prevalence and causes of nocturia. J Urol. 2010;184(2):440-46

30. Chen TC, Parker JD, Clark J, Shin HC, Rammon JR, Burt VL. National Health and Nutrition Examination Survey: estimation procedures, 20112014. Vital Health Stat 2. 2018;(177):1-26.

31. Asplund R, Aberg H. Nocturnal micturition, sleep and well-being in women of ages 40-64 years. Maturitas. 1996;24(1-2):73-81.

32. Weiss JP, Blaivas JG, Jones M, Wang JT, Guan Z, Study G. Age-related pathogenesis of nocturia in patients with overactive bladder. J Urol. 2007;178(2):548-51.
33. Onukwugha E, Zuckerman IH, McNally D, Coyne KS, Vats V, Mullins CD The total economic burden of overactive bladder in the United States: a disease-specific approach. Am J Manag Care. 2009;15(4 Suppl):S90-97.

34. Ganz ML, Smalarz AM, Krupski TL, et al. Economic costs of overactive bladder in the United States. Urology. 2010;75(3):526-32.

35. Greenberg PE, Fournier AA, Sisitsky T, Pike CT, Kessler RC. The economic burden of adults with major depressive disorder in the United States (2005 and 2010). J Clin Psychiatry. 2015;76(2):155-62

36. American Diabetes Association. Economic costs of diabetes in the U.S. in 2017. Diabetes Care. 2018;41(5):917-28.

37. Robinson D, Suman S. Managing nocturia: the multidisciplinary approach. Maturitas. 2018;116:123-29.

38. Weiss JP, van Kerrebroeck PE, Klein BM, Norgaard JP. Excessive nocturnal urine production is a major contributing factor to the etiology of nocturia. J Urol. 2011;186(4):1358-63.

39. Cornu JN, Abrams P, Chapple CR, et al. A contemporary assessment of nocturia: definition, epidemiology, pathophysiology, and management-a systematic review and meta-analysis. Eur Urol. 2012;62(5):877-90.

40. Leggett LE, Khadaroo RG, Holroyd-Leduc J, et al. Measuring resource utilization: a systematic review of validated self-reported questionnaires. Medicine (Baltimore). 2016;95(10):e2759. 
The Economic Burden of Nocturia on the U.S. Health Care System and Society:

A National Health and Nutrition Examination Survey Analysis

\begin{tabular}{|c|c|c|c|c|}
\hline \multicolumn{5}{|c|}{$\begin{array}{l}\text { Sensitivity Analysis (Nocturia Defined as } \geq 1 \text { Voids/Night)-HRU, Work Productivity, and Health } \\
\text { Status Stratified by Age }\end{array}$} \\
\hline \multirow[b]{2}{*}{ Study Outcome } & \multicolumn{4}{|c|}{ Comparison of Individuals with vs. Without Nocturia ( $\geq 1$ vs. 0 Voids/Night) } \\
\hline & \multicolumn{2}{|c|}{$\begin{array}{l}\text { Total Sample } \\
(\mathrm{N}=22,300)\end{array}$} & \multicolumn{2}{|c|}{$\begin{array}{l}\text { Excluding Individuals with CHF/Diabetes } \\
\qquad(\mathrm{n}=19,107)\end{array}$} \\
\hline HRU & $\operatorname{IRR}(95 \% \mathrm{CI})^{\mathrm{a}}$ & \multirow{2}{*}{$P$ Value } & $\operatorname{IRR}(95 \% \mathrm{CI})^{\mathrm{a}}$ & \multirow{2}{*}{$\begin{array}{c}P \text { Value } \\
0.002\end{array}$} \\
\hline Hospitalization & $1.26 \quad(1.10,1.43)$ & & $1.28 \quad(1.10,1.49)$ & \\
\hline Aged $20-44$ years & $1.30(1.06,1.59)$ & 0.011 & $1.31 \quad(1.06,1.61)$ & 0.012 \\
\hline Aged $45-64$ years & $1.16 \quad(0.93,1.45)$ & 0.191 & $1.19 \quad(0.89,1.58)$ & 0.233 \\
\hline Aged $65+$ years & $1.08 \quad(0.87,1.35)$ & 0.487 & $1.08 \quad(0.80,1.45)$ & 0.606 \\
\hline Outpatient visits & $1.11 \quad(1.07,1.15)$ & $<0.001$ & $1.12(1.08,1.17)$ & $<0.001$ \\
\hline Aged $20-44$ years & $1.11 \quad(1.05,1.17)$ & $<0.001$ & $1.12 \quad(1.06,1.19)$ & $<0.001$ \\
\hline Aged $45-64$ years & $1.09 \quad(1.02,1.17)$ & 0.008 & $1.10 \quad(1.02,1.19)$ & 0.011 \\
\hline Aged $65+$ years & $1.08 \quad(1.02,1.14)$ & 0.014 & $1.09 \quad(1.01,1.18)$ & 0.021 \\
\hline Work Productivity & Difference $(95 \% \mathrm{CI})^{\mathrm{b}}$ & $P$ Value & Difference $(95 \% \mathrm{CI})^{\mathrm{b}}$ & $P$ Value \\
\hline Weekly hours worked & $-1.90(-2.81,-0.98)$ & $<0.001$ & $-1.99(-2.97,-1.02)$ & $<0.001$ \\
\hline Aged $20-44$ years & $-2.07(-3.18,-0.96)$ & $<0.001$ & $-2.07 \quad(-3.20,-0.94)$ & $<0.001$ \\
\hline Aged 45-64 years & $-2.53(-4.18,-0.89)$ & 0.003 & $-2.59(-4.30,-0.87)$ & 0.004 \\
\hline \multirow[t]{2}{*}{ Aged $65+$ years } & $0.03 \quad(-1.47,1.53)$ & 0.971 & $-0.33(-2.11,1.45)$ & 0.713 \\
\hline & OR $(95 \% \mathrm{CI})^{\mathrm{c}}$ & $P$ Value & OR $(95 \% \mathrm{CI})^{\mathrm{c}}$ & $P$ Value \\
\hline Employment status & $0.84 \quad(0.77,0.92)$ & $<0.001$ & $0.83 \quad(0.76,0.91)$ & $<0.001$ \\
\hline Aged $20-44$ years & $0.79 \quad(0.70,0.89)$ & $<0.001$ & $0.79 \quad(0.69,0.90)$ & $<0.001$ \\
\hline Aged $45-64$ years & $0.80 \quad(0.67,0.95)$ & 0.014 & $0.81 \quad(0.68,0.97)$ & 0.021 \\
\hline Aged $65+$ years & $1.03 \quad(0.76,1.38)$ & 0.863 & $0.90 \quad(0.68,1.21)$ & 0.486 \\
\hline Current Health Status & OR $(95 \% \mathrm{CI})^{\mathrm{d}}$ & $P$ Value & OR $(95 \% \mathrm{CI})^{\mathrm{d}}$ & $P$ Value \\
\hline Very good or excellent & $0.68 \quad(0.62,0.74)$ & $<0.001$ & $0.66 \quad(0.60,0.73)$ & $<0.001$ \\
\hline Aged 20-44 years & $0.72 \quad(0.62,0.83)$ & $<0.001$ & $0.71 \quad(0.61,0.82)$ & $<0.001$ \\
\hline Aged $45-64$ years & $0.59 \quad(0.50,0.71)$ & $<0.001$ & $0.57 \quad(0.46,0.70)$ & $<0.001$ \\
\hline Aged $65+$ years & $0.85 \quad(0.67,1.08)$ & 0.178 & $0.83 \quad(0.62,1.10)$ & 0.196 \\
\hline \multicolumn{5}{|c|}{$\begin{array}{l}\text { aAn IRR > } 1 \text { indicates that individuals with nocturia had more hospitalizations or outpatient visits compared with individuals without nocturia. } \\
b_{A} \text { difference }<0 \text { indicates that individuals with nocturia worked fewer hours weekly compared with individuals without nocturia. } \\
{ }^{c} \text { An odds ratio }<1 \text { indicates that individuals with nocturia are less likely to report being employed compared with individuals without nocturia. } \\
{ }^{d} \text { An odds ratio < } 1 \text { indicates that individuals with nocturia are less likely to report being in very good or excellent health compared with individuals without nocturia. } \\
C H F=\text { congestive heart failure; } C I=\text { confidence interval; } H R U \text { = health care resource utilization; IRR=incidence rate ratio; OR=odds ratio. }\end{array}$} \\
\hline
\end{tabular}


The Economic Burden of Nocturia on the U.S. Health Care System and Society:

A National Health and Nutrition Examination Survey Analysis

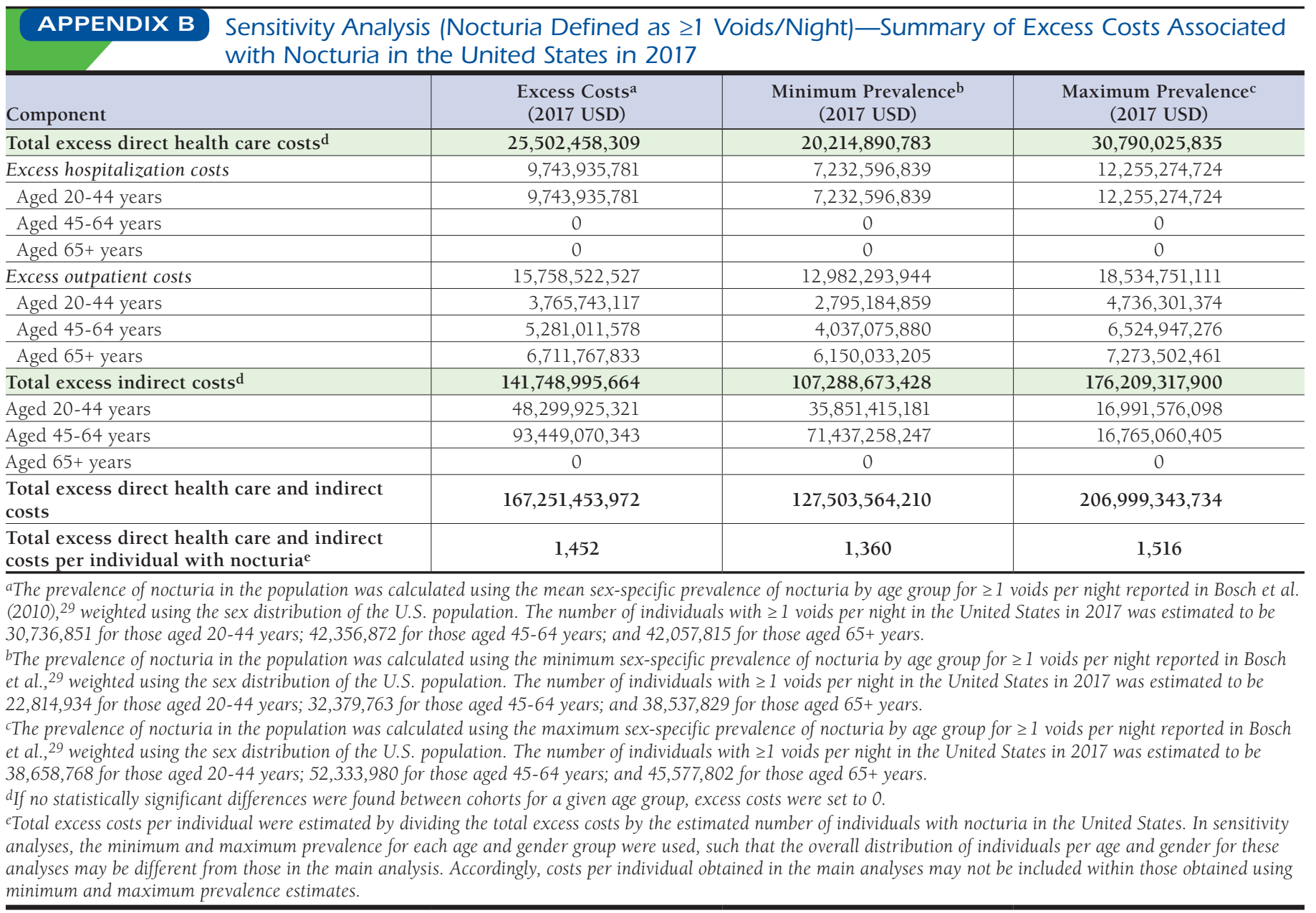

